\title{
Selective attention as tuning: The case of stroke weight
}

\author{
JUDITH AVRAHAMI \\ Hebrew University of Jerusalem, Jerusalem, Israel
}

\begin{abstract}
It has long been demonstrated that when grouping occurs, attention transfer between grouped elements is facilitated, as compared with attention transfer between elements - similarly distant—-that are not grouped. This has been shown for grouping by connectedness, by orientation, and by color. The present article extends these findings to the case of similarity in coarseness. By using spatial cuing to elements drawn with different strokes, it is shown that the visual processing of elements that share stroke heaviness with the cued element is more efficient than that of elements that do not. Three experiments, in which cue validity regarding the target's location and/or its stroke is manipulated, show that the facilitation has both an endogenous and an exogenous component. The findings are discussed in terms of visual tuning to the features of a stimulus, with tuning being the initial stage of visual processing required for identification and discrimination. It is proposed that grouping, rather than explaining the facilitation observed, can be explained by the notion of visual tuning to features. The findings also point to potential methodological pitfalls when different stroke weights are used, unintentionally, in visual displays.
\end{abstract}

For a long time, attention was regarded as a tuning to the location and size of the relevant stimulus. Such spatial tuning was first modeled by a spotlight - to account for the specificity of location - and then by a zoom lens - to integrate location and size - and its characteristics have been studied extensively (B. A. Eriksen \& C. W. Eriksen, 1974; C. W. Eriksen \& St. James, 1986; Posner, 1980). It was soon realized, however, that spatial tuning does not account for all the phenomena related to attentional efficiency and that other characteristics of the scene play an important role in visual processing, and the notion of tuning has been practically forsaken.

\section{Effects of Scene Characteristics on Attention}

As an example of the effects of scene characteristics, Kahneman and Henik (1981) have demonstrated that attention can shift more easily between items that belong to the same group than between equidistant items that belong to different groups. Kramer and Jacobson (1991) have shown that in the judging of a target, lines sharing a color with a target line or lines that are connected to it may interfere more than equidistant lines that do not. Similarly, Baylis and Driver (1992) have shown that letters that share a color with or are connected by lines (even if dashed) to the target letter interfere more than equidistant letters that do not.

In an attempt to incorporate the effects of scene characteristics under a single conceptualization, it has been

Correspondence concerning this article should be addressed to J. Avrahami, Goldie Rotman Center for Cognitive Science and Education and Center for the Study of Rationality, Hebrew University of Jerusalem, Jerusalem, Israel (e-mail: judav@vms.huji.ac.il). suggested that attention selects whole objects that are present in the scene - that is, that attention is object based (Duncan, 1984; Egly, Driver, \& Rafal, 1994). The study of attention under this conceptualization has motivated a rich volume of research, and a variety of experimental paradigms have been perfected (see Scholl, 2001, for a comprehensive review). As a result, it is now widely accepted that when attention is drawn to a stimulus, other stimuli that belong to the same object receive priority over stimuli that do not.

Still, many students of attention would admit that the concept of object often has to be greatly stretched if it is to accommodate all the existing data. For example, it has been shown that the sharing of shade or texture (e.g., G. Davis, Driver, Pavani, \& Shepherd, 2000; Kramer \& Watson, 1996), orientation (e.g., Avrahami, 1999, Experiment 1), or their combination (e.g., Lavie \& Driver, 1996), also affects the ease with which attention is shifted from one location to another. At the same time, the expected effects of objects are sometimes absent even when simple, straightforward objects are used (e.g., Avrahami, 1999, Experiment 2). In order to loosen the concept of object, some researchers - prominently, Kramer and his colleagues - have advocated the notion of grouped array, instead of that of objects, invoking Gestalt principles of grouping to determine attentional groups. This conceptualization can, indeed, accommodate a number of findings that cannot be explained by the concept of object. However, more recent work has shown that even grouping does not account for all the phenomena related to the efficiency of attentional processing. Thus, for example, Cepeda and Kramer (1999) have shown that task-relevant organization may also have an effect on efficiency; Han, Dosher, and Lu (2003) have shown that the number of 
task-relevant dimensions (two of one object and one or two of two objects) is a more important determinant of efficiency than is the number of objects involved. The present article reports a study that was designed to test the effect of yet another feature on the efficiency of attention shifts between locations.

The feature studied here is the coarseness of the elements in the display - namely, the weight of the stroke with which they are drawn. The effect of stroke weight on attention has, to the best of my knowledge, never been studied before, although it obviously shares aspects both with size and with spatial frequency. For example, in the most well-known demonstration of grouping by similarity in size, in which rows of dots group into lines due to the similarity in dot size, size could also be conceived of as the dots' coarseness. Similarly, when advance preparation intervenes in the detection of a particular spatial frequency (e.g., E. T. Davis, 1981; Schyns \& Oliva, 1999), it could result from preparation for the coarseness, or stroke, of the lines differing in spatial frequency. It has been shown before that both size and spatial frequency are related to the efficiency of visual processing. When the visual system has to switch between sizes (e.g., Bundesen \& Larsen, 1975; Farell \& Pelli, 1993) or between spatial frequencies (e.g., E. T. Davis, Kramer, \& Graham, 1983; Solomon \& Pelli, 1994), processing is compromised. However, the role played by coarseness cannot be teased out from these studies: When letters changed in size, they also changed in stroke, and even when the stimuli consisted of gratings, it is not clear whether it was the width of the lines in the grating, the distance between them, or both that was responsible for the effects observed.

The present study addressed coarseness separately, keeping the size of the stimuli constant. It consisted of three experiments in which the costs of switching between elements that differed in stroke were explored.

\section{The Present Study}

The study had a number of objectives: First, it was intended as a demonstration that the coarseness of elements is another characteristic of a scene that can affect attention shifts over a display. This was achieved by showing that when stroke was shared by two stimuli, attention transfer between them was expedited. Second, the demonstration has some urgent methodological import, since it points out potential pitfalls of using, unawares, stimuli of different stroke in studies of visual attention. Finally, the study adds to the growing list of findings that call for relaxing the concepts of both object-based attention and groupedarray-based attention, advocating a return to the notion of attention as tuning to account for the existing body of data. Attention is conceived of here as the initial stage of visual processing required for identification and discrimination.

The experimental paradigm used here was that of spatial cuing, introduced by Egly et al. (1994). In this paradigm, attention is cued to one location in space, and then a target appears either in the cued location or in one of two equidistant neighboring locations. The stimulus in one of the neighboring locations shares some feature with the stimu- lus in the cued location, whereas that in the other does not. By comparing the costs of attention shift to the two uncued locations, one can learn the effects of the shared feature. The paradigm was used originally to study the effect of being part of the same object and revealed that attention is more easily shifted to a location that belongs to the same rectangle as the cued location than to a location that belongs to another (Egly et al., 1994). The paradigm has often been employed since to find the boundary conditions of such facilitation (e.g., Abrams \& Law, 2000; Avrahami, 1999; Cepeda \& Kramer, 1999; Goldsmith \& Yeari, 2003; Lamy \& Egeth, 2002). Here, it was used to study the effects of the sharing of stroke weight.

The study consisted of three experiments differing in the information value of an exogenous cue. In the first experiment, the cue was informative of target location and corresponded, with high probability, to target stroke. In the second, it was not informative of location but still corresponded to target stroke more often than not. In the third experiment, the cue was again uninformative of location, but its correspondence to target stroke was manipulated, corresponding with a high probability in one condition and with a low probability in another.

\section{EXPERIMENT 1}

Four (digital) number 8 figures were displayed-one above, one below, one to the right, and one to the left of screen center. Two of these figures had a heavy stroke, and two had a light stroke. They were always arranged so that each figure had one neighbor with the same stroke and another with a different one. After a short adaptation period, one of the four figures flickered, and then one of them turned into either a 2 or a 5. The participants' task was to detect what figure it turned into (see Figure 1). In most cases, the 8 figure that changed was the one that had flickered, but in the remaining cases it was one of its two neighbors.

\section{Method}

Participants. Twenty-four students at the Hebrew University, half of them male and half female, participated in the experiment as paid volunteers.

Materials and Stimuli. The stimuli were four digital 8 figures, two drawn with a 1-pixel line (light stroke) and two drawn with a 4-pixel line (heavy stroke). For the participants, seated approximately $60 \mathrm{~cm}$ from the computer monitor, the line subtended either $0.03^{\circ}$ or $0.12^{\circ}$ of visual angle (corresponding to approximately 16 and 4 cycles per degree). The figures were $4 \times 8 \mathrm{~mm}$, subtending $0.38^{\circ} \times 0.76^{\circ}$ of visual angle. The figures were drawn at the four ends of a virtual rhombus centered on the screen, so that each was located $3.82^{\circ}$ from the center. All four possible arrangements of light- and heavy-stroke figures were used, under the constraint that each figure had one similar and one different neighbor. After a cuing flicker and a short interval, two line segments were omitted from one of the figures, turning it into either the 2 figure or the 5 figure.

Procedure and Design. A trial began with the appearance of the four 8 figures, which stayed on the screen for $450 \mathrm{msec}$; then one of the figures disappeared for $30 \mathrm{msec}^{2}$ and was redrawn ${ }^{1}$; finally, after another $195 \mathrm{msec}$, one of the figures turned into a 2 or a 5 . This display remained in view until the participant responded by pressing a key with the left hand for 2 or another key with the right hand for 5 . 


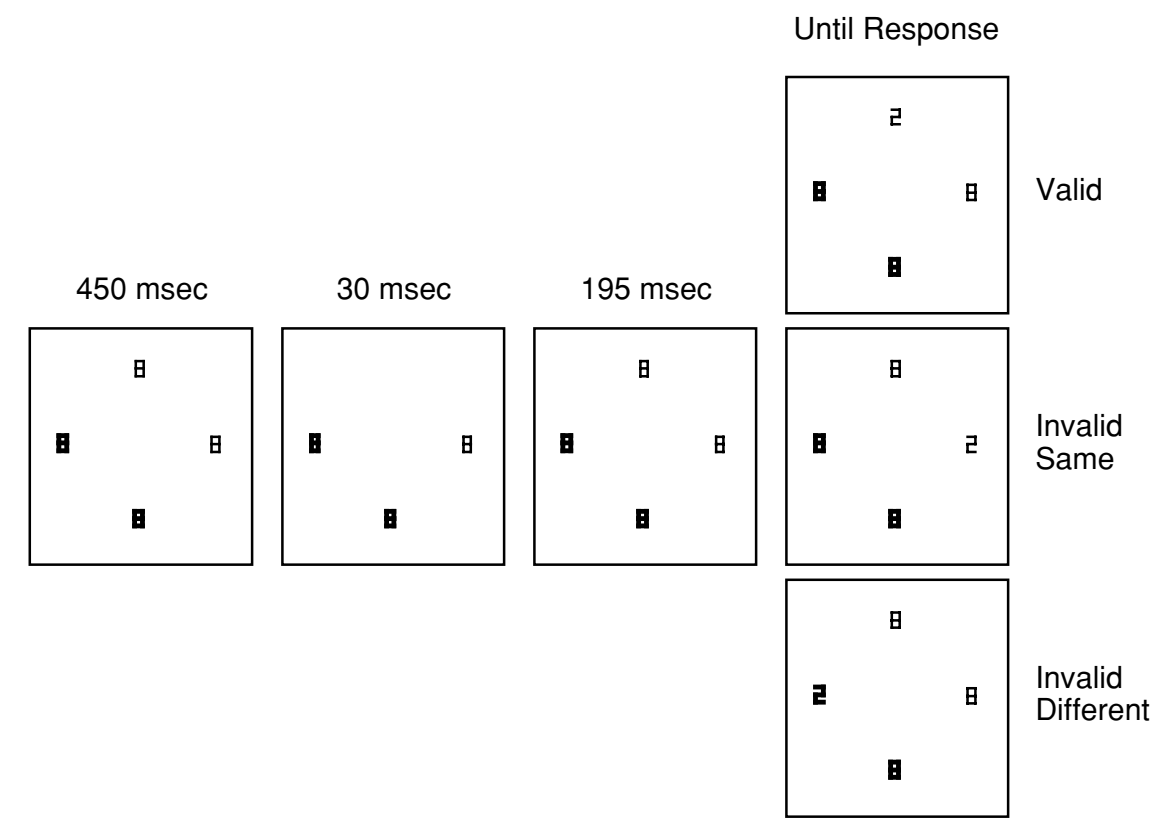

Figure 1. The experimental sequence for one of the arrangements of stimuli with 2 as the target stimulus.

A 675 -msec blank preceded the beginning of the next trial. Every participant performed three blocks of 64 trials each. A practice block of 24 trials preceded these experimental blocks. Feedback on accuracy was provided only at the end of a block.

There were four arrangements of the light- and heavy-stroke figures, and the cue was equally likely at all four locations. The target appeared with a probability of $2 / 3$ in the cued location (valid trials) and with a probability of $1 / 6$ in each of its two neighboring locations (invalid trials). Thus, in half of the invalid trials, the target shared stroke with the cued figure (invalid same-stroke trials), and in the other half it differed in stroke from the cued figure (invalid differentstroke trials). The trials were presented in random order.

Thus, the variables manipulated were validity (whether the target appeared at the cued location or at a neighboring location) and, within the invalid trials, stroke sameness (whether the target had the same stroke as the cued figure or not). A resulting additional variable was target stroke (whether the target figure was of a light or a heavy stroke).

The experiment was run on a Mac LCIII computer with a 13-in. monitor and was controlled by the VScope software package (Enns \& Rensink, 1992)

\section{Results and Discussion}

Median response time (RT), for correct trials only, was calculated for every participant. The mean of these RTs was $507 \mathrm{msec}$ for valid trials and $677 \mathrm{msec}$ for invalid trials. This highly significant difference of $170 \mathrm{msec}$ $\left[F(1,23)=262.20, M S_{\mathrm{e}}=2,649, p<.001\right]$ replicates the well-known effect of location cuing on spatial resolution. The analysis also revealed a main effect of target stroke: Responding to heavy-stroke targets was faster by $13 \mathrm{msec}$ than responding to light-stroke ones $[F(1,23)=4.66$, $\left.M S_{\mathrm{e}}=814, p=.042\right]$.

Most important for the issue at hand is the difference between the two types of invalid trials (stroke sameness). A second analysis compared RTs for invalid same-stroke and invalid different-stroke trials. When the invalid target and the cued figure were of the same stroke, the RT was $657 \mathrm{msec}$, whereas when the invalid target differed from the cued figure in line stroke, the RT was $697 \mathrm{msec}$. The difference of $40 \mathrm{msec}$ is highly significant $[F(1,23)=$ $\left.37.19, M S_{\mathrm{e}}=1,489, p<.001\right]$. The effect of target stroke did not reach significance in this analysis $[F(1,23)=1.85$, $\left.M S_{\mathrm{e}}=2,166, p=.187\right]$, and there was no interaction of stroke sameness with target stroke $(F<1)$.

Accuracy was high and was not affected by the experimental manipulation of stroke sameness $(F<1)$. Even the effect of validity on accuracy did not reach significance $\left[F(1,23)=2.79, M S_{\mathrm{e}}=0.0008, p=.108\right]$.

The results are clear, demonstrating that when attention is cued to a stimulus of a certain stroke, switching to another stimulus of the same stroke is more efficient than switching to an equidistant stimulus of a different stroke.

The results of Experiment 1, although clear-cut with respect to the effects of stroke on visual processing, leave one question unanswered. Apparently, when the visual system selects a stimulus, it adjusts to the stimulus' features for optimal processing - in the present case, to its stroke. When it has to rapidly select a new stimulus that differs in stroke, a costly readjustment is required. It is as yet unclear whether this adjustment — namely, this tuning to stroke weight - is voluntary, reacting to the informativeness of the cue, or involuntary, reacting to its flickering. Even if voluntary, it is as yet unclear whether it is because the cued figure would most likely be the target figure (given the informativeness of the cue) or because the stroke of the cued figure corresponded, more often than not, to that of the target figure. To answer this question, two additional experiments were conducted. In both, 
the cue was uninformative of target location, but the stroke of the target was either more or less likely to be the same as that of the cued figure.

\section{EXPERIMENT 2}

Experiment 2 was designed to test whether the cost of switching between the different strokes of the cued and the invalidly cued different-stroke target figure, shown in Experiment 1, would also be observed when the cue was uninformative of target location. If the tuning was involuntary, the cost of retuning should be similar in the present experiment to that observed before, since the layout and the flicker of the cued figure are the same here. If, on the other hand, tuning was voluntary or if it was a combination of the two, the cost would be expected to diminish, if related to cue predictiveness of target coarseness, or even disappear, if related to cue predictiveness of target location. To increase the power of the test, in order to be able to conclude not only that there was an effect (if one was found), but also that there was none (if none was found), the number of participants in the second experiment was doubled.

\section{Method}

The second experiment was identical to the first, with the following exceptions.

Participants. Forty-eight students at the Hebrew University, half of them males and half females, participated in this experiment. None had participated in the first experiment.

Design. The probability that the cued figure would be the target (the one changed into a 2 or a 5 ) was reduced from $2 / 3$ to $1 / 3$ and was thus equal to the probability that each of its two neighbors would be the target.

The number of trials in the practice block was 48 .

\section{Results and Discussion}

The same analyses as those conducted in Experiment 1one testing for the effect of cue validity and the second testing for the effect of stroke sameness of invalid targetswere conducted here. As will be presented below, the results of the second experiment replicated those of the first, with some interesting differences.

The effect of validity on speed was still very strong RTs were $552 \mathrm{msec}$ for valid and $646 \mathrm{msec}$ for invalid trials $\left[F(1,47)=165.91, M S_{\mathrm{e}}=2,556, p<.001\right]$-and so was the effect of target stroke [with responses to targets with the heavier stroke being processed $23 \mathrm{msec}$ faster; $\left.F(1,47)=31.42, M S_{\mathrm{e}}=817, p<.001\right]$. The effect of stroke sameness was also still highly significant: RT was $639 \mathrm{msec}$ for invalid same-stroke targets and $654 \mathrm{msec}$ for invalid different-stroke targets $\left[F(1,47)=10.52, M S_{\mathrm{e}}=\right.$ $966, p=.002]$. Stroke sameness again did not interact with target stroke $(F<1)$. Note, however, that the effect of stroke sameness had now diminished to $15 \mathrm{msec}$.

The effect of validity on accuracy, although small, reached significance this time $\left[F(1,47)=4.31, M S_{\mathrm{e}}=0.0005, p=\right.$ $.043]$; but as before, there was no effect of either stroke sameness or of target stroke on accuracy (both $F_{\mathrm{s}}<1$ ).

The results of both experiments demonstrate the cost of switching between stroke weights. At the same time, the effects are clearly not identical, since the cost of switching was $40 \mathrm{msec}$ in the first experiment but only $15 \mathrm{msec}$ in the second. A new analysis, this time for the data of both experiments combined, with cue informativeness as a between-participants variable, revealed strong interactions between cue informativeness (i.e., experiment) and both validity (i.e., whether the target appeared in the cued figure or not) and stroke sameness (i.e., whether the invalidly cued target was of the same or a different stroke) $\left[F(1,70)=35.82, M S_{\mathrm{e}}=2,587, p<.001\right.$, and $F(1,70)=$ $9.17, M S_{\mathrm{e}}=1,138, p=.003$, for the interaction between cue informativeness and validity and for the interaction between cue informativeness and stroke sameness, respectively]. These results are presented in Figure 2.

The differences between the two experiments demonstrate that tuning to stroke is not purely involuntary. Had it been so, no difference would have been found between the two experiments, since there was no difference in the exogenous nature of the cue in the two experiments. Since a difference was found, a voluntary component was obviously at work. There is good reason why this component was of greater magnitude in the first than in the second experiment: In Experiment 1 the stroke of the cued figure was predictive of the stroke of the target on five sixths of the trials, whereas in Experiment 2 it was predictive of it on four sixths of the trials. This reduction in predictiveness in Experiment 2 can explain the reduction in the effect of stroke sameness observed there. Still, it is unclear whether the smaller effect observed in Experiment 2 was voluntary, due to the likely correspondence between the stroke of the cue and that of the target, or whether it reflected the remaining involuntary component of tuning to the stroke of the flickering figure. Experiment 3 was conducted to determine which of these two possibilities was more viable.

\section{EXPERIMENT 3}

In this experiment, in addition to the four figure arrangements in Experiments 1 and 2, in which each figure had one neighbor with the same stroke and another with a different one, two new arrangements were added, in which spatial frequency alternated between neighbors. These arrangements consisted either of ones in which the upper and lower figures were light in stroke and the left and right figures were heavy in stroke or of ones in which the upper and lower figures were heavy in stroke and the left and right figures were light in stroke. As a result, in these new arrangements, the stroke of every figure differed from that of both its neighbors; hence, the stroke of the cued figure matched that of the target in only one third of the cases in these new arrangements (only in the validly cued targets), as compared with two thirds in the original ones. The question was whether targets whose stroke differed from that of the cued target but had the majority stroke in the triad of candidate targets (in the new arrangements) would be processed more efficiently than targets whose stroke differed from that of the cued target but had the minority stroke in the triad (in the original arrangements). 


\section{Effects of Validity and Spatial Frequency on Speed}

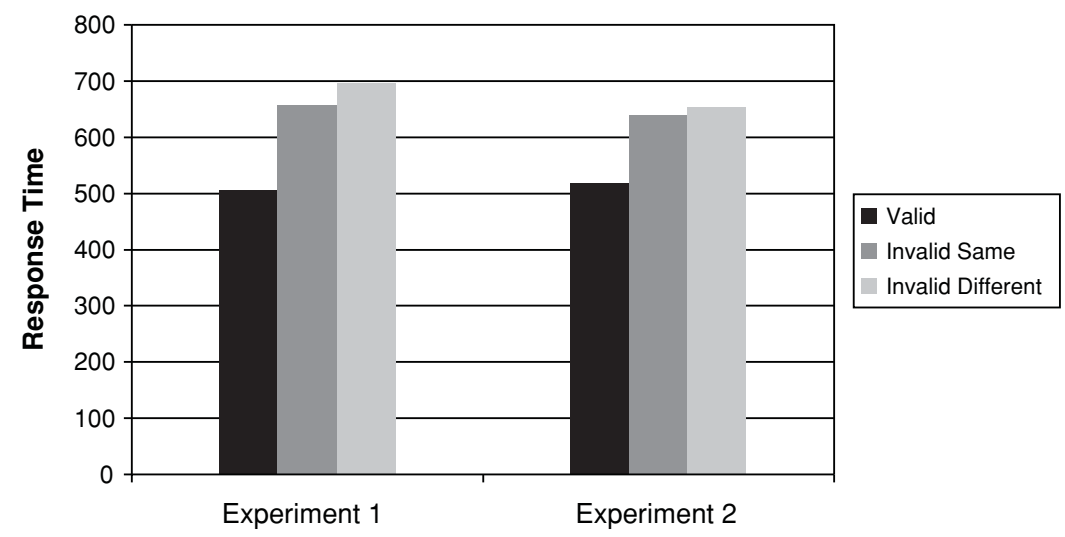

Figure 2. The effects of cue validity and similarity in spatial frequency on speed in both Experiments 1 and 2.

\begin{abstract}
Method
Participants. Twenty-four students at the Hebrew University, half of them male and half female, participated in this experiment. None had participated in the previous experiments.

Materials, Procedure, and Design. The materials, procedure, and design were identical to those in Experiment 2, with the following exceptions.

Two new arrangements were added, as has been explained above, for a total of six arrangements. This resulted in having, for each of the four screen locations, 6 valid trials, 4 invalid trials in which the invalidly cued target had the same stroke as that of the cued figure, 4 trials in which the invalidly cued target had a different stroke from that of the cued target but that was the stroke of the majority of stimuli in the triad of target candidates, and 4 trials in which the invalidly cued target had a different stroke from that of the cued target and its stroke was the minority in the triad. The participants performed two blocks of 72 trials each, preceded by a practice block also of 72 trials.
\end{abstract}

\section{Results and Discussion}

The analyses were similar to those conducted for Experiments 1 and 2 , with the exception that here there were three types of invalidly cued targets: (1) targets that shared stroke with the cued figure (and had the majority stroke in the triad), (2) targets that did not share stroke with the cued figure but had the majority stroke in the triad, and (3) targets that did not share stroke with the cued figure and had the minority stroke in the triad.

The effect of validity on speed was again very strongRTs were $519 \mathrm{msec}$ for valid and $654 \mathrm{msec}$ for invalid trials $\left[F(1,23)=93.22, M S_{\mathrm{e}}=4,680, p<.001\right]$-and so was the effect of target stroke [with responses to heavystroke targets being faster by $11 \mathrm{msec} ; F(1,23)=10.21$, $\left.M S_{\mathrm{e}}=280, p=.004\right]$. Looking separately at each of the three invalidly cued trial conditions, we see that RTs were $638 \mathrm{msec}$ for the same-stroke (and majority) trials, $663 \mathrm{msec}$ for the different-but-majority-stroke trials, and $661 \mathrm{msec}$ for the different-and-minority-stroke trials. An analysis of the invalidly cued trials revealed a significant effect only of the type of trial $\left[F(2,46)=5.36, M S_{\mathrm{e}}=\right.$ $1,789, p=.008]$. As the means indicate, this effect was due only to the difference between the same stroke and the different stroke, and not to the majority-minority aspect of the target's stroke.

As in the previous experiments, although target stroke had a significant effect, it did not interact with the different conditions of invalidly cued trials $(F<1)$. Accuracy again was not affected by the main experimental manipulation.

The lack of effect of the majority-minority manipulation shows that the cost of shifting attention to a target that differs in stroke from that of a cued figure- shown in Experiments 2 and 3-was due to involuntary tuning to the stroke of the cued figure. Had the cost shown in Experiment 2 resulted from the fact that the stroke of the cued figure was also the stroke of the majority-hence, worthwhile voluntarily tuning to-it would have disappeared in the minority condition in Experiment 3.

\section{GENERAL DISCUSSION}

The results of the three experiments indicate that the visual system tunes even to the stroke of the attendedstimulus lines. Hence, when the visual system tunes to the stroke of a stimulus, the availability of other stimuli depends on their own stroke: They are more available when they are similar to the one tuned to than when they differ from it. The present research thus is an extension of previous studies of attention shifts between locations. The results also show that such tuning has both a voluntary and an involuntary component. Thus, tuning can be more effective when the probability that it serves the very stimulus to be processed is high and less effective when the probability is low.

One might be concerned that the stimuli that differed in stroke also differed in luminance, with the figure drawn with a heavier stroke producing a stronger contrast than did the other. However, even if this were the case, prior research has shown that the visual system cannot tune to contrast in the way it does to spatial frequency or loca- 
tion (e.g., E. T. Davis et al., 1983), always favoring higher contrast instead. Advance preparation for the contrast of an element in a display would thus have no effect. In other words, if contrast had been the cause of the results, one would expect a cost when the usual system switched between high- and low-contrast elements, but not when it switched between low- and high-contrast elements. The lack of an interaction between target stroke and stroke sameness indicates that this was not the case.

\section{Implications of the Results \\ for Other Cuing Experiments}

The experimental paradigm used here is the one that has been used mainly to study the conditions under which attention is object based. The present results may, therefore, shed new light on previous studies that looked for, but did not always find, effects of objects on attention. To demonstrate this point, I shall use notions of coarsenesssensitive attention shifts, advanced here, to reinterpret two studies testing for the presence and absence of object-based advantages with exogenous versus endogenous cuing (Goldsmith \& Yeari, 2003; Macquistan, 1997).

Macquistan (1997), using a paradigm similar to that in Egly et al. (1994) with either an exogenous or an endogenous cue, found a same-object advantage when one end of one rectangle was cued exogenously, but not when it was cued endogenously. Goldsmith and Yeari (2003) replicated Macquistan's results when using the exact same procedure but did get the same-object advantage when they used a nonvisual (auditory) endogenous cue or when they instructed the participants to not focus attention on the visual endogenous cue.

It is interesting to note that both Macquistan (1997) and Goldsmith and Yeari (2003, Experiment 1B) used, as endogenous cue, a line segment that differed from the cued objects in line stroke. Thus, if the participants tuned to the endogenous cue, turning attention to the rectangular objects would require retuning to a new line stroke. This may be the reason why the participants apparently completely skipped the tuning to the rectangles, searching directly for the target; hence, no same-object advantage has been observed in that condition. Moreover, it is quite likely that when Goldsmith and Yeari instructed their participants not to focus on the endogenous cue, they instructed them, in effect, not to tune to the cue but to keep focusing on the rectangles - that is, on their line stroke. In that case, the visual endogenous cuing, like the nonvisual one, did enable tuning to the rectangular objects, resulting in the reemergence of the same-rectangle advantage.

The discussion above of Macquistan (1997) and of Goldsmith and Yeari (2003) is by no means a criticism of their work. It is used only to demonstrate how taking into account the coarseness of stimuli can provide a new perspective on old findings. I have no doubt that many experimental findings that have failed to demonstrate object-based effects and have, therefore, remained unpublished could be reanalyzed and reconsidered in light of the sensitivity of attentional processes to coarseness.

\section{Related Work on Tuning to Spatial Frequency}

As was mentioned in the introduction, there is some similarity between the study reported here and the body of research demonstrating the relation between visual attention and spatial frequency (e.g., Yeshurun \& Carrasco, 2000). Much work has been devoted to assessing the degree to which advance preparation for a specific spatial frequency enhances detection, with advance preparation operationalized in various manners. One is by manipulating the certainty with which the upcoming spatial frequency of the stimulus can be known in advance (E. T. Davis, 1981; E. T. Davis et al., 1983; Hübner, 1996; Ohtani \& Mori, 2002; Sowden, Özgen, Schyns, \& Daoutis, 2003), by validly precuing the upcoming spatial frequency of the target stimulus (E. T. Davis et al., 1983; Hübner, 1996; Ohtani \& Mori, 2002; Sowden et al., 2003) or by invalidly cuing it (Sowden et al., 2003). Effects of the nature of the cue and of its location were further explored by Hübner (1996). That body of research revealed that advance knowledge of the upcoming stimulus' spatial frequency enhances detection both through certainty and through precuing. What is more, wrong expectations (through invalid precuing) have been found to result in worse performance than does a lack of expectations.

Another way in which advance preparation has been operationalized is by priming a stimulus of a certain spatial frequency by stimuli of similar or different spatial frequencies. ${ }^{2}$ For example, Tanaka and Sagi (2000) studied the priming effect of lateral stimuli on the detection of a central one and found that the effect is related to the similarity in spatial frequency between priming stimuli and target. Similarly, Schyns and Oliva (1999) demonstrated that practice with one spatial frequency determines which spatial frequency observers will pick when stimuli of two spatial frequencies are superimposed.

Other demonstrations of the specificity of visually processing different spatial frequencies can be found in studies that involved concurrent processing of stimuli with either similar or different spatial frequencies. Examples in which the task required concurrent processing of two stimuli are Adini and Sagi (1992) and Shulman and Wilson (1987a, 1987b); examples in which the task required filtering one of two stimuli are Solomon and Pelli (1994) and Majaj, Pelli, Kurshan, and Palomares (2002). Concurrent processing was easier when the stimuli shared spatial frequency, and filtering was easier when they did not.

All these studies have indicated the sequentiality of tuning to spatial frequency, demonstrating both a voluntary and an involuntary component of that tuning. In that, their findings are in line with those of the present study. Unlike the work on spatial frequency, where stroke of lines and the distance between lines covary, the results reported here are more specific, showing that the weight of lines requires specific tuning even when the distance between them is kept constant.

\section{Summary}

One way to summarize the results is to say, simply, that they present a new variation on grouping by similarity. 
Demonstrating grouping by similarity in stroke, they expand on previous findings in which stroke was correlated with size or, more specifically, in which the weight of lines was correlated with the distance between them.

Another way to summarize the results is as further indication of the specificity of visual selection. It is generally assumed that when attention selects a stimulus, it addresses each dimension separately (e.g., color, shape, or size) - hence, the inefficiency of visually searching for targets specified on two different dimensions (e.g., Treisman \& Gelade, 1980). The present results indicate that attention may separately select each value of a dimension. Consequently, stimuli whose feature values are not tuned to are less available for attended processing. As such, they do not interfere in the processing of objects that do share the features to which attention is tuned. This can also explain the efficiency of visually searching for targets that differ from their distractors in color (i.e., differ by their value on a dimension). By the same token, tuning to a specific orientation can explain not only the effects of unconnected lines in the display on attention transfer (Avrahami, 1999), but also the very phenomenon of texture segregation (Ben-Shahar, Scholl, \& Zucker, 2006), where effects similar to those of object-based attention have been found for regions segregated by the orientation of their grain.

There is obviously no conflict between the two summary options, and one could easily be reduced to the other. The question is which notion has a higher explanatory value. With grouping being the elder notion, visual tuning is often subsumed under grouping. However, it is no secret that the principles of grouping are not explanations of visual phenomena but descriptions of them, describing the emergence of object candidates. The stimuli sharing stroke in the present study, being more than $5^{\circ}$ of visual angle apart, were hardly candidates for a single object or even for a single grouped array. I therefore wish to take this opportunity and suggest that the more common reduction direction should be reversed: Rather than saying that grouping explains facilitation in switching between similar stimuli, say that sequential tuning to features is the basis for grouping. Since alternating between big and small, coarse and fine, red and blue, and so forth can only be done sequentially, only some of the stimuli are available for optimal processing at every instant. For stimuli to be available at the same instant $i$ s to be grouped.

The present research shows that the search for features that attention tunes to has by no means been exhausted. Thus, the questions addressed in the classical work of Treisman (e.g., Treisman \& Gormican, 1988) and Julesz (e.g., Julesz, 1981) have not been fully answered yet. It is my belief that we must know these features to constrain any future theory of visual processing.

\section{REFERENCES}

Abrams, R. A., \& Law, M. B. (2000). Object-based visual attention with endogenous orienting. Perception \& Psychophysics, 62, 818-833.

AdINI, Y., \& SAGI, D. (1992). Parallel processes within the "spot-light" of attention. Spatial Vision, 6, 61-77.
Avrahami, J. (1999). Objects of attention, objects of perception. Perception \& Psychophysics, 61, 1604-1612.

BaYLIS, G. D., \& Driver, J. (1992). Visual parsing and response competition: The effect of grouping factors. Perception \& Psychophysics, 51, 145-162.

Ben-Shahar, O., Scholl, B. J., \& Zucker, S. W. (2006). Bridging the gap between object-based attention and texton-based segmentation: How attention flows through orientation-defined textures. Manuscript submitted for publication.

Bundesen, C., \& Larsen, A. (1975). Visual transformation of size. Journal of Experimental Psychology: Human Perception \& Performance, 1, 214-220.

Cepeda, N. J., \& Kramer, A. F. (1999). Strategic effects on objectbased attentional selection. Acta Psychologica, 103, 1-19.

DAVIS, E. T. (1981). Allocation of attention: Uncertainty effects when monitoring one or two visual gratings of noncontiguous spatial frequencies. Perception \& Psychophysics, 29, 618-622.

Davis, E. T., Kramer, P., \& Graham, N. (1983). Uncertainty about spatial frequency, spatial position, or contrast of visual patterns. Perception \& Psychophysics, 33, 20-28.

Davis, G., Driver, J., Pavani, F., \& ShePherd, A. (2000). Reappraising the apparent costs of attending to two separate visual objects. Vision Research, 40, 1323-1332.

Duncan, J. (1984). Selective attention and the organization of visual information. Journal of Experimental Psychology: General, 113, 501517

EgLy, R., Driver, J., \& RAFAL, R. D. (1994). Shifting visual attention between objects and locations: Evidence from normal and parietal lesion subjects. Journal of Experimental Psychology: General, 123, 161-177.

ENNS, J. T., \& RENSINK, R. A. (1992). VScope: A general purpose tachistoscope for the Macintosh. Vancouver: Micropsych Software.

Eriksen, B. A., \& Eriksen, C. W. (1974). Effects of noise letters upon the identification of a target letter in a nonsearch task. Perception \& Psychophysics, 16, 143-149.

ERIKSEN, C. W., \& ST. James, J. D. (1986). Visual attention within and around the field of focal attention: A zoom lens model. Perception \& Psychophysics, 40, 225-240.

Farell, B., \& Pelli, D. G. (1993). Can we attend to large and small at the same time? Vision Research, 33, 2757-2772.

Goldsmith, M., \& Yeari, M. (2003). Modulation of object-based attention by spatial focus under endogenous and exogenous orienting. Journal of Experimental Psychology: Human Perception \& Performance, 29, 897-918.

Han, S., Dosher, B. A., \& Lu, Z.-L. (2003). Object attention revisited: Identifying mechanisms and boundary conditions. Psychological Science, 14, 598-604.

HüBNER, R. (1996). The efficiency of different cue types for reducing spatial-frequency uncertainty. Vision Research, 36, 401-408.

Julesz, B. (1981). Textons, the elements of texture perception, and their interactions. Nature, 290, 91-97.

Kahneman, D., \& HeniK, A. (1981). Perceptual organization and attention. In M. Kubovy and J. R. Pomerantz (Eds.), Perceptual organization (pp. 181-211). Hillsdale, NJ: Erlbaum.

Kramer, A. F., \& Jacobson, A. (1991). Perceptual organization and focused attention: The role of objects and proximity in visual processing. Perception \& Psychophysics, 50, 267-284.

Kramer, A. F., \& Watson, S. E. (1996). Object-based visual selection and the principle of uniform connectedness. In A. F. Kramer, M. G. H. Coles, \& G. D. Logan (Eds.), Converging operations in the study of visual selective attention (pp. 395-414). Washington, DC: American Psychological Association.

Lamy, D., \& Egeth, H. (2002). Object-based selection: The role of attentional shifts. Journal of Experimental Psychology: Human Perception \& Performance, 64, 52-66.

Lavie, N., \& Driver, J. (1996). On the spatial extent of attention in object-based visual selection. Perception \& Psychophysics, 58, 1238 1251

Macquistan, A. D. (1997). Object-based allocation of visual attention in response to exogenous, but not endogenous, spatial precues. Psychonomic Bulletin \& Review, 4, 512-515.

Majaj, N. J., Pelli, D. G., Kurshan, P., \& Palomares, M. (2002). 
The role of spatial frequency channels in letter identification. Vision Research, 42, 1165-1184.

OHTANI, Y., \& MoRI, S. (2002). Spatial-frequency uncertainty and cuing effects on psychometric functions for contrast detection. Psychonomic Bulletin \& Review, 9, 270-277.

Posner, M. I. (1980). Orienting of attention. Quarterly Journal of Experimental Psychology, 32, 3-25.

ScHOLL, B. J. (2001). Objects and attention: The state of the art. Cognition, 80, 1-46.

Schyns, P. G., \& Oliva, A. (1999). Dr. Angry and Mr. Smile: When categorization flexibly modifies the perception of faces in rapid visual presentations. Cognition, 69, 243-265.

Shulman, G. L., \& Wilson, J. (1987a). Spatial frequency and selective attention to local and global information. Perception, 16, 89-101.

Shulman, G. L., \& Wilson, J. (1987b). Spatial frequency and selective attention to spatial location. Perception, 16, 103-111.

Solomon, J. A., \& Pelli, D. G. (1994). The visual filter mediating letter identification. Nature, 369, 395-397.

Sowden, P. T., Özgen, E., Schyns, P. G., \& Daoutis, C. (2003). Expectancy effects on spatial frequency processing. Vision Research, 43, 2759-2772.
TANaKa, Y., \& SAGI, D. (2000). Attention and short-term memory in contrast detection. Vision Research, 40, 1089-1100.

Treisman, A., \& Gelade, G. (1980). A feature-integration theory of attention. Cognitive Psychology, 12, 97-136.

Treisman, A., \& Gormican, S. (1988). Feature analysis in early vision: Evidence from search asymmetries. Psychological Review, 95, 15-58. Yeshurun, Y., \& CARRasco, M. (2000). The locus of attentional effects in texture segmentation. Nature Neuroscience, 3, 622-627.

\section{NOTES}

1. This method of cuing was chosen in order not to introduce any new shades, orientations, or spatial frequencies.

2 . In the studies cited so far, precuing has often been symbolicwhether visual or auditory - with the exception of the iconic cuing in Hübner (1996), which can be regarded as priming.

(Manuscript received March 17, 2004; revision accepted for publication April 20, 2005.) 